\title{
Formação de hábitos alimentares na primeira infância: benefícios da alimentação
}

\section{Saudável}

\author{
Formation of eating habits in early childhood: benefits of healthy eating \\ Formación de hábitos alimentarios en la primera infancia: beneficios de una alimentación \\ saludable
}

Recebido: 06/11/2021 | Revisado: 12/11/2021 | Aceito: 16/11/2021 | Publicado: 25/11/2021

\author{
Neurani Rodrigues Araújo \\ ORCID: https://orcid.org/0000-0002-2519-2809 \\ Centro Universitário Fametro, Brasil \\ E-mail: neuraaraujo6@gmail.com \\ Francisca Marta Nascimento de Oliveira Freitas \\ ORCID: https://orcid.org/0000-0002-0044-0925 \\ Centro Universitário Fametro, Brasil \\ E-mail: Francisca.freitas@fametro.edu.br \\ Rosimar Honorato Lobo \\ ORCID: https://orcid.org/0000-0002-8954-2302 \\ Centro Universitário Fametro, Brasil \\ E-mail: rosimar.lobo@fametro.edu.br
}

\begin{abstract}
Resumo
Introdução: Os hábitos alimentares na primeira infância devem ser observados do começo ao fim, visto que o acompanhamento nutricional da criança promoverá o melhor desenvolvimento do seu organismo e corpo nessa fase primordial. Objetivo Geral: elucidar hábitos alimentares na primeira infância. Metodologia: Para a realização desse estudo optou-se por uma pesquisa na modalidade de revisão bibliográfica dedutiva. Resultado e Discussão: Do ponto de vista nutricional o Aleitamento Materno Exclusivo (AME) consiste no mais nutritivo e adequado alimento para a criança até os seis primeiros meses de vida, por ser rico em vitaminas, proteínas, carboidratos, gorduras, sais minerais e água. Conclusão: Além de entender o papel do nutricionista no desenvolvimento da criança, deve ser observada a importância do envolvimento dos pais nessa fase, dada pelo valor da educação nutricional sendo abordada desde cedo e levada pelos pequenos para o resto da vida, influenciando a formação dos hábitos alimentares na vida adulta e levando isso de geração em geração.
\end{abstract}

Palavras-chave: Hábitos alimentares; Primeira infância; Educação nutricional.

\begin{abstract}
Introduction: Eating habits in early childhood must be observed from beginning to end, as the nutritional monitoring of the child will promote the best development of their organism and body in this primordial phase. General Objective: elucidate eating habits in early childhood. Methodology: To carry out this study, we opted for a research in the form of a deductive bibliographic review. Results and Discussion: From a nutritional point of view, Exclusive Breastfeeding (EBF) is the most nutritious and adequate food for a child up to the first six months of life, as it is rich in vitamins, proteins, carbohydrates, fats, minerals and water. Conclusion: In addition to understanding the role of the nutritionist in the child's development, the importance of parental involvement at this stage should be noted, given the value of nutrition education being addressed from an early age and taken by the little ones for the rest of their lives, influencing the formation of eating habits in adult life and taking it from generation to generation.
\end{abstract}

Keywords: Eating habits; Early childhood; Nutrition education.

\section{Resumen}

Introducción: Los hábitos alimentarios en la primera infancia deben observarse de principio a fin, ya que el seguimiento nutricional del niño favorecerá el mejor desarrollo de su organismo y cuerpo en esta fase primordial. Objetivo general: dilucidar los hábitos alimentarios en la primera infancia. Metodología: Para la realización de este estudio se optó por una investigación en forma de revisión bibliográfica deductiva. Resultados y Discusión: Desde el punto de vista nutricional, la Lactancia Materna Exclusiva (LME) es el alimento más nutritivo y adecuado para un niño hasta los primeros seis meses de vida, ya que es rico en vitaminas, proteínas, carbohidratos, grasas, minerales y agua. Conclusión: Además de comprender el papel del nutricionista en el desarrollo del niño, conviene señalar la importancia de la participación de los padres en esta etapa, dado el valor de que la educación nutricional sea abordada 
desde temprana edad y tomada por los más pequeños para el resto de la jornada. sus vidas, influyendo en la formación de hábitos alimentarios en la vida adulta y llevándolos de generación en generación.

Palabras clave: Hábitos alimenticios; Niñez temprana; Educación nutricional.

\section{Introdução}

O consumo de alimentos ultra processados tem um impacto prejudicial na saúde das crianças devido ao alto teor de açúcar, sódio e gordura, gerando um inadequado consumo de micronutrientes, este comportamento são práticas alimentares não saudáveis (Brekke et al., 2007; Jaime et al., 2018).

A importância dos hábitos alimentares desenvolvidos na infância é notória, visto que é nessa primeira fase que ocorre a introdução alimentar que pode determinar os padrões alimentares em anos subsequentes. A formação do paladar das crianças possui componente genético e ambiental/social, que pode influenciar precocemente o padrão de alimentação que a criança irá reproduzir no transcorrer dos anos (Beauchamp \& Mennella 2009; Madruga et al., 2012).

Na pesquisa de Lopes et al. (2017) é ressaltada a importância dos profissionais da saúde no acompanhamento de famílias e aconselhamento para a boa alimentação nos primeiros estágios de vida dos seus filhos de forma que destaque a influência positiva do aleitamento materno e desencorajamento da introdução de outros tipos de leite, bem como a forma correta de incluir a alimentação complementar.

A ajuda dos pais nessa fase da infância é de suma importância, os pais devem incentivar os filhos a manterem hábitos saudáveis como fazerem atividade física e comerem alimentos naturais, principalmente legumes, frutas e verduras logo nos primeiros anos de vida. Deve-se evitar alimentos ricos em sódio, gorduras e açúcares, apesar de serem nutrientes essenciais para o desenvolvimento saudável da criança, quando consumidos em excesso tornam-se inimigos mortais da saúde (Yokota et al., 2010; Bastos et al., 2019, p.128).

O acompanhamento familiar é imprescindível para estimular a criança na busca de hábitos de vida saudáveis e o seu papel como núcleo apoiador para o enfretamento da obesidade e de suas repercussões negativas (Victorino et al., 2014)

No início da alimentação pode acontecer erros como a oferta inadequada de alimentos, e é importante que haja na hora da alimentação uma separação dos alimentos no prato, para que a criança consiga distinguir as cores, sabores, aromas e texturas dos alimentos oferecidos a ela, e com isso verificar se há algum que não foi aceito por ela (Ramos, 2000; Ramos Alves, 2014).

Segundo Oliveira et al., (2017) afirma que os lactentes que o desenvolvimento neuropsicomotor e menor incidência de hábitos orais deletérios foram melhor desenvolvidos possuem grande influência do aleitamento materno, ainda que não sofra alterações ou diferenças ao introduzir alimentos com textura líquida e pastosa.

A criança suas preferências alimentares, e a família tem o importante papel junto com a escola de incentivar hábitos alimentares mais saudáveis, no entanto, fatores genéticos e hereditários podem interferir nesses hábitos, ou quando a criança muda de ambiente e começa a frequentar locais que influenciam na alimentação como creches e escolas (Ferreira, 2018).

Em sua pesquisa Monteiro e Costa (2004); Cunha (2014) mencionam que "Uma boa alimentação é aquela que mantém o organismo em estado de saúde, ou seja, com osso e dentes fortes, peso e estatura de acordo com o biótipo do indivíduo, boa disposição, resistência às enfermidades, vontade de trabalhar e divertir se, para isso se faz necessária uma dieta balanceada que contenha variados nutrientes com múltiplas funções."

A alimentação na primeira infância é um fator básico no desenvolvimento da criança, manter hábitos alimentares saudáveis favorece o crescimento físico, fisiológico e intelectual da criança desde a gestação, levando este desenvolvimento para o resto da vida, além de proteger o organismo contra infecções e fornecendo nutrientes fundamentais para a formação de todos os sistemas do corpo. 
Sendo assim, o interesse nesta área surgiu através do impacto que a formação dos hábitos alimentares pode proporcionar nos primeiros anos de vida, onde as primeiras experiências nutricionais, da primeira infância, são capazes de programar o padrão metabólico por toda vida determinando uma maior ou menor propensão a desenvolver diferentes patologias.

O objetivo geral deste estudo é analisar a importância dos hábitos alimentares, desde a primeira infância, de um indivíduo e a influência desses hábitos a curto e longo prazo, enfatizando a importância do acompanhamento nutricional, sendo os objetivos específicos: explicar a importância da amamentação nos primeiros meses de vida, identificar fatores que atuam na formação dos hábitos alimentares da infância, identificar os malefícios de uma alimentação inadequada na infância e explicar a influência dos pais no processo de educação nutricional na primeira infância.

\section{Metodologia}

O estudo será realizado com coleta de dados a partir de fontes secundarias, por meio de levantamento bibliográfico e baseado na experiência vivenciada por autores. Para a organização dos dados coletados se fara uso da metodologia dedutiva, pois segundo Marconi e Lakatos (2010) o processo dedutivo parte de uma constatação geral para uma especifica, do processo maior para o menor.

Para elaboração da pesquisa, foram realizados levantamentos bibliográficos fazendo o uso de materiais publicados em forma de revistas, órgãos oficiais, artigos em sites como SCIELO (Scientific Eletronic Library), PubMed (Serviço da National Library of Medicine) e Biblioteca Virtual de Saúde (BVS).

Após as buscas através do cruzamento dos descritores nas bases indexadas: "Nutrição materno-infantil", "Introdução alimentar", "Aleitamento materno exclusivo", "Nutrição infantil", "Obesidade infantil", "Desnutrição infantil", foram resultados um total de 1.124 estudos.

Dos estudos resultantes, um total de 523 foram encontrados na base de dados Scielo, dos quais apenas 245 apresentavam o texto completo disponível, e 67 foram publicados nos últimos dez anos, resultando após análise crítica um total de 10 artigos que respondem à questão norteadora desse estudo.

Ao que se refere à base de dados Lilacs, após o cruzamento dos descritores foram encontrados um total de 426 estudos, dos quais apenas 197 estudos apresentavam o texto completo e disponível, e apenas 57 estudos foram publicados nos últimos dez anos obedecendo os critérios previamente estabelecidos, respondendo assim, após uma análise crítica 6 artigo à questão norteadora desse estudo.

Ao que se refere à base de dados Lilacs, após o cruzamento dos descritores foram encontrados um total de 175 estudos, dos quais apenas 103 estudos apresentavam o texto completo e disponível, e apenas 47 estudos foram publicados nos últimos dez anos obedecendo os critérios previamente estabelecidos, respondendo assim, após uma análise crítica 4 artigo à questão norteadora desse estudo.

Dessa forma, totalizaram um total de 20 estudos que iram compor esse estudo, os quais após análise crítica constatouse que respondem à questão norteadora do estudo aqui proposto, apresentados a partir do corpus desse estudo. 
Quadro 1. Corpús do Estudo.

\begin{tabular}{|c|c|c|c|c|}
\hline N\% ANO & TITULO DO ESTUDO & PERIÓDICO & AUTORES & BASE DE DADOS \\
\hline A1/ 2020 & $\begin{array}{c}\text { Determinantes Socioeconômicos E De Saúde } \\
\text { Da Desnutrição Infantil: Uma Análise Da } \\
\text { Distribuição Espacial }\end{array}$ & Saúde e pesquisa. & $\begin{array}{l}\text { Garcia, L., \& Roncalli, } \\
\text { A. }\end{array}$ & SCIELO \\
\hline A2/ 2019 & $\begin{array}{l}\text { Auto eficácia na amamentação exclusiva: } \\
\text { avaliação dos domínios técnica e pensamentos } \\
\text { intrapessoais em puérperas. }\end{array}$ & $\begin{array}{l}\text { Enfermagem em } \\
\text { foco. }\end{array}$ & Lima, C., et al. & LILACS \\
\hline $\mathrm{A} 3 / 2019$ & $\begin{array}{c}\text { O Papel Do Ambiente Obesogênico E Dos } \\
\text { Estilos De Vida Parentais No Comportamento } \\
\text { Alimentar Infantil. }\end{array}$ & Rev Paul Pediatr & Dantas, R., \& Silva, G. & SCIELO \\
\hline A4/ 2017 & $\begin{array}{l}\text { Influência do comportamento dos pais durante a } \\
\text { refeição e no excesso de peso na infância Escola } \\
\text { Anna Nery }\end{array}$ & $\begin{array}{c}\text { Revista de } \\
\text { Enfermagem. }\end{array}$ & Melo, K.M., et al. & SCIELO \\
\hline A5/2017 & $\begin{array}{l}\text { A importância nutricional da alimentação } \\
\text { complementar. }\end{array}$ & $\begin{array}{l}\text { Revista Ciências } \\
\text { Nutricionais Online, }\end{array}$ & Oliveira, M., et al. & LILACS \\
\hline $\mathrm{A} 6 / 2016$ & Infant feeding: beyond the nutritional aspects. & J Pediatr & Silva, G., et al. & PUBMED \\
\hline A7/2015 & $\begin{array}{l}\text { Fatores que influenciam na interrupção do } \\
\text { aleitamento materno exclusivo em nutrizes. }\end{array}$ & $\begin{array}{l}\text { Revista Gaúcha de } \\
\text { Enfermagem }\end{array}$ & Amaral, L., et al. & LILACS \\
\hline A8/2015 & Obesidade Infantil: Causas e Consequências. & $\begin{array}{l}\text { Eletronic Journal of } \\
\text { Pharmacy }\end{array}$ & Nascimento, G., et al. & LILACS \\
\hline A9/2013 & $\begin{array}{l}\text { Conhecimentos maternos: influência na } \\
\text { introdução da alimentação complementar. }\end{array}$ & Com. Ciência Saúde & $\begin{array}{l}\text { Haack, A. \& Martins, } \\
\text { M. }\end{array}$ & SCIELO \\
\hline A10/2013 & $\begin{array}{l}\text { Prevalência e fatores associados à desnutrição e } \\
\text { ao excesso de peso em menores de cinco anos } \\
\text { nos seis maiores municípios do Maranhão. }\end{array}$ & $\begin{array}{l}\text { Revista Brasileira } \\
\text { Epidemiol. }\end{array}$ & Chagas, D., et al. & LILACS \\
\hline A11/2013 & $\begin{array}{c}\text { Motivos do sucesso da amamentação exclusiva } \\
\text { na perspectiva dos pais. }\end{array}$ & $\begin{array}{c}\text { Revista eletrônica de } \\
\text { enfermagem }\end{array}$ & Cabral, P., et al. & SCIELO \\
\hline A12/2013 & $\begin{array}{l}\text { Obesidade: Aspectos Epidemiológicos E } \\
\text { Prevenção. }\end{array}$ & Rev Med. & Carvalho, E., et al. & SCIELO \\
\hline A13/2013 & $\begin{array}{l}\text { Aleitamento materno, a introdução da } \\
\text { alimentação complementar e sua relação com a } \\
\text { obesidade infantil. }\end{array}$ & $\begin{array}{l}\text { Revista Brasileira de } \\
\text { Obesidade, Nutrição } \\
\text { e Emagrecimento } \\
\end{array}$ & VICARI, E. & LILACS \\
\hline A14/2012 & Avaliação do Estresse na Obesidade Infantil. & PSICO & $\begin{array}{c}\text { Bertoletti, J., \& } \\
\text { Garcia-Santos,S. C. }\end{array}$ & SCIELO \\
\hline A15/2012 & $\begin{array}{c}\text { Obesidade Infantil: Estudo em Crianças Num } \\
\text { ATL. }\end{array}$ & Revista de nutrição & $\begin{array}{l}\text { Pereira, P., \& Lopes, } \\
\text { L. }\end{array}$ & PUBMED \\
\hline A16/2012 & $\begin{array}{l}\text { A Relação Entre A Desnutrição e o } \\
\text { Desenvolvimento Infantil. }\end{array}$ & $\begin{array}{l}\text { Rev. Assoc. Bras. } \\
\text { Nutr. }\end{array}$ & Fraga, J., et al. & SCIELO \\
\hline A17/2012 & Alimentação na primeira infância no Brasil. & Revista APS & Lima, D., et al. & PUBMED \\
\hline A18/2011 & $\begin{array}{c}\text { Crianças Desnutridas: Percepção Da Família } \\
\text { Quanto Ao Cuidado. }\end{array}$ & Cienc Cuid Saude. & Frota, M. A., et al. & PUBMED \\
\hline A19/2011 & $\begin{array}{l}\text { Introdução de alimentos industrializados e de } \\
\text { alimentos de uso tradicional na dieta de crianças } \\
\text { de creches públicas no município de São Paulo. }\end{array}$ & Revista de nutrição & Toloni, M., et al. & SCIELO \\
\hline A20/2011 & Obesidade Infantil na Percepção dos Pais. & Rev Paul Pediatr, & $\begin{array}{l}\text { Tenorio, A., \& } \\
\text { Cobayashi, F. }\end{array}$ & SCIELO \\
\hline
\end{tabular}

Fonte: Autores.

\section{Resultados e Discussão}

\subsection{Aleitamento Materno Exclusivo (AME)}

Do ponto de vista nutricional o Aleitamento Materno Exclusivo (AME) consiste no mais nutritivo e adequado alimento para a criança até os seis primeiros meses de vida, por ser rico em vitaminas, proteínas, carboidratos, gorduras, sais minerais e água. Esses nutrientes são essenciais para o crescimento e desenvolvimento infantil (Amaral et al., 2015)

Segundo Gallo et al., (2008) apud Martins e Santana (2013) "Ao escolher o aleitamento materno exclusivo, a mãe provê alimento ao filho, promove a sua saúde e fortalece o contato afetivo, vínculo que se inicia na concepção, cresce durante a gestação e se fortalece com a amamentação".

O acompanhamento pré-natal, as consultas puerperais e as consultas de acompanhamento do crescimento e desenvolvimento da criança parecem contribuir sobremaneira para o sucesso na manutenção do aleitamento materno exclusivo até os seis meses de vida (Lima et al., 2019). 
Para complementarem sua pesquisa, Rosa e Delgado (2017) mencionaram a revisão integrativa de Demitto et al. (2010) a respeito das orientações sobre amamentação na assistência pré-natal:

O aleitamento materno exclusivo nos primeiros 6 meses de vida do bebê é de suma importância, pois garante nutrição adequada, ajuda no desenvolvimento das estruturas orais, como lábios, língua, bochechas, palato duro e mole, responsáveis pelo funcionamento adequado das funções de respiração, sucção, mastigação, deglutição e fonoarticulação, além de propiciar o padrão de respiração nasal (Demitto et al., 2010)

Segundo Cabral et al. (2013) “A alegria, o prazer, a realização pessoal, o estresse, o sofrimento e a dor fizeram parte da teia de emoções/dificuldades dos casais que vivenciaram a amamentação”, portanto, além da interação mãe e filho, é possível observar a diversidade de sensações entre os demais envolvidos no processo de amamentação.

Lima et al. (2012) Destaca que:

"No Brasil, os trabalhos nesta área, têm se limitado, quase exclusivamente, à questão da prática do aleitamento materno, indiscutivelmente, de fundamental importância, nos primeiros 24 meses de vida. Porém, há outros elementos que envolvem a alimentação e nutrição neste período, que merecem, de forma semelhante, atenção especial. Por exemplo, os aspectos qualitativos e quantitativos da alimentação artificial e o próprio processo de desmame." (Lima et al., 2012, p. 336).

De acordo com Haak e Martins (2013) “A introdução adequada e no tempo oportuno da alimentação complementar traz inúmeras vantagens para a saúde da criança e para a formação de seus hábitos alimentares, assim como a continuação do aleitamento materno até os dois anos de idade, mantendo os fatores de proteção", destacando a importância da introdução alimentar de forma adequada para a criança.”

De acordo com sua publicação sobre alimentação infantil, Silva et al., (2016) destacam que:

Não só o que a criança come é importante, como também como, quando, onde e quem a alimenta. Cada vez mais tem sido dada a devida importância à interação entre a pessoa que alimenta a criança e ela. Essa interação deve resultar na chamada alimentação responsiva, cabe ao cuidador a responsabilidade de ser sensível aos sinais da criança e aliviar tensões durante a alimentação, além de fazer das refeições momentos agradáveis; enquanto é papel da criança expressar os sinais de fome e saciedade com clareza e ser receptiva às tentativas de alimentação (Silva et al., 2016, p. 56).

Segundo Vicari (2013) “A nutrição no início da vida afeta não apenas o desenvolvimento cerebral, crescimento e composição corporal, mas também a programação metabólica com impacto sobre as doenças crônicas do adulto relacionadas com a alimentação.”

É necessário reforçar os cuidados nos primeiros meses de vida das crianças, pois nestes elas encontram-se mais sensíveis pela fase de crescimento e dependentes dos cuidados da mãe. Para que as necessidades nutricionais dos lactentes sejam atendidas, é importante que ele receba aleitamento materno exclusivo até o sexto mês de idade, depois deve-se introduzir os alimentos complementares para suprir suas necessidades energéticas (Oliveira \& Avi, 2017, p.36).

De acordo com Meller (2014); Melo et al., (2017) "No Brasil, um estudo realizado com 4.388 crianças com idade inferior a cinco anos mostrou que a prevalência de excesso de peso foi de 6,6\%". Segundo Alves \& Cunha (2020) "A boa alimentação deve tornar-se, portanto, um hábito desde criança por toda a vida, pois é fundamental no desenvolvimento da criança, por isso é preciso conscientizar sobre os benefícios que nos trará desde a infância.” 
Segundo Coutinho et al., (2008); Figueiredo (2015) "Em vista dos frequentes debates acerca da transição nutricional que se vivencia nas últimas décadas, na qual se observa a redução dos índices de desnutrição ao mesmo tempo em que se assiste prevalências crescentes de obesidade.”

De acordo com Black et al. (2008); Barros (2020) "A desnutrição engloba um conjunto de situações patológicas que resultam de uma alimentação insuficiente, comprometendo a saúde, o desenvolvimento e o crescimento da criança."

[...] as consequências da introdução e da utilização precoce e incorreta de alimentos industrializados poderão ser amenizadas por iniciativas de educação nutricional, que atinjam parcela significativa da população (Toloni et al., 2011, p. 69)

A inter-relação entre hábitos alimentares, manutenção da saúde e desenvolvimento de doenças crónicas não transmissíveis, tem vindo a tornar-se cada vez mais evidente nos últimos trinta anos, dado o reconhecimento de que as características da dieta podem exercer influência decisiva sobre o estado de saúde dos indivíduos (WHO, 1990; Aparício, 2016).

[...] O papel dos pais na obesidade infantil é também muito importante, devido à hereditariedade e ao exemplo que os progenitores dão em casa, que influencia o comportamento das crianças, através da alimentação e dos hábitos de atividade física (Pereira \& Lopes, 2011).

A obesidade infantil é um processo dinâmico, no qual o comportamento, a cognição a regulação emocional interage mutuamente, sofrendo ainda a influência das atitudes parentais, atividade física, padrão nutricional e estresse familiar (Puder \& Munsch, 2010; Bertoletti \& Garcia-Santos, 2012).

A obesidade pode causar alterações que afetam praticamente todos os sistemas do corpo humano e alcançar proporções extensas a longo prazo como crescimento precoce, problemas ósseos e musculares (postura incorreta e osteoartrose), doenças de pele (tumores e estrias), problemas no aparelho urinário e genital (falta de ovulação e gestações problemáticas), gastrointestinais (colecistite e hérnia hiatal) e outros (Nascimento, 2015).

[...]O desmame precoce, a existência de sobrepeso na família, a alimentação excessiva e distúrbio na dinâmica familiar, além da redução da prática de atividades físicas, são fatores comumente associados à sua etiologia (Carvalho et al., 2013).

Embora se verifiquem algumas relações, na amostra, entre os hábitos alimentares e a obesidade: as crianças com obesidade almoçam mais na escola e menos em casa, vão mais a locais com fast-food, costumam comer menos vegetais nas suas refeições e comem menos vezes sopa, verificando-se também que comem menos doces, elas não são estatisticamente significativas (Pereira et al., 2011).

De acordo com Lacerda (2003); Chagas et al. (2013) “A desnutrição é caracterizada como uma condição patológica decorrente da falta de energia e proteínas, em variadas proporções, podendo ser agravada por infecções repetidas"

A ansiedade, a preocupação e o medo diante da situação de saúde das crianças foram observados. A família não reconhece a verdadeira origem multicausal da desnutrição infantil, mas mostra culpa e tristeza diante da incapacidade de fornecer condições de vida à criança (Frota et al., 2011).

A avaliação do crescimento infantil, além de um indicador de desnutrição pregressa, é uma medida indireta da qualidade de vida da população, visto que a maioria dos problemas de saúde e nutrição durante a infância de relacionam com o consumo alimentar inadequado e infecções de repetição, os quais estão relacionados com o padrão de vida da população (Neves, 2016; Garcia \& Roncalli, 2020). 
A desnutrição em uma família pode ter múltiplas causas e significados, mas não pode passar despercebida para a sociedade, que tem o dever de cuidar de suas crianças e protegê-las, sobretudo suprindo suas necessidades mais básicas (Fraga \& Varela, 2012).

A "má-nutrição", comumente resulta da combinação de dietas inadequadas e infecção. O consumo de alimentos incapazes de prover as calorias imprescindíveis, atrelado à ingestão de valor menor que quatro tipos de grupos alimentares pelas crianças são fortes preditores de desnutrição" (Rodrigues, 2001; BJSCR, 2013-2014).

De acordo com Toloni et al., (2011):

Conclui que "Além de conscientizar os pais sobre a idade adequada e a forma correta de introdução de alimentos, é necessário alertá-los sobre os malefícios causados pela introdução errônea e precoce dos industrializados, considerando os fatores culturais, comportamentais e afetivos envolvidos com a alimentação."

Segundo Lima et al., (2011) "Como qualquer mulher que vivencia a maternagem, a jovem mãe irá desenvolver um papel fundamental em relação ao cuidado com o filho, sendo o cuidado com a alimentação um dos mais importantes, haja vista as repercussões que determina na saúde do infante.”

Segundo Silva (2016); Dantas e Silva (2019) "Os pais devem estimular a criança a comer com moderação, levando-a a associar o início de uma refeição à sensação de fome e o término da refeição à sensação de saciedade".

Em sua pesquisa, Tenório e Cobayashi (2011) concluem que boa parte de estudos que abordam a obesidade promovem a recomendação da alimentação saudável e prática de atividades físicas, porém, é necessário atentar-se à percepção dos pais seja levada em consideração para enfrentar a situação com seus filhos e profissionais.

De acordo com Epstein (2003); Coelho et al (2014) "Parece ser consonante na comunidade científica que a família será o principal mediador social no desenvolvimento e prevenção da obesidade, quer seja pelo estímulo para a prática de atividades físicas, pela oferta acertada de alimentos e modelagem de comportamentos, quer seja pelo ajustamento emocional face à comida."

\section{Conclusão}

A formação de hábitos alimentares na primeira infância é de suma importância para que a saúde seja reforçada nessa fase tão importante de desenvolvimento da criança, como também influencia nos hábitos da família e, principalmente, da mãe que fornece a primeira fonte de alimentação ainda na fase de amamentação. A relação dos pais com essa busca por orientação e aconselhamento nutricional é uma garantia de melhor qualidade para seus filhos, prevenindo-os de desenvolver patologias e remediando possíveis situações desconfortáveis com relação a saúde de suas crianças.

A falta de informação traz essa comodidade, devido ao que uns compartilham com os outros a respeito da alimentação de forma saudável, visto que a falta de orientação nutricional estimula o pensar da população de que se reeducar é uma possibilidade cada vez mais distante, por questões financeiras e outros fatores.

Ainda há muito o que ser estudado com relação à busca por métodos eficazes de levar a educação nutricional para a população em geral e, principalmente, para gestantes de baixa renda que não tem acesso ao que é disponibilizado pelo governo para auxiliar tanto em fornecimento de leite (como exemplo o programa Leite do Meu Filho, que tem como objetivo fornecer alimentação de crianças em situações de vulnerabilidade) e também em diversos programas de fornecimento de orientação nutricional e de recursos para auxiliar na disponibilização de alimentação para as crianças e famílias. 


\section{Referências}

Alves, G., et al. (2020). A Importância Da Alimentação Saudável Para O Desenvolvimento Humano. Revista Perspectivas Online: Humanas \& Sociais Aplicadas, 10(27), 46-62. <https://ojs3.perspectivasonline.com.br/humanas_sociais_e_aplicadas/article/view/1966/1661>.

Alves, G. Alimentação Complementar De Crianças Na Primeira Infância E A Influência Da Mídia. Tese (graduação no curso de nutrição) - Centro Universitário de Brasília (UniCEUB), Brasília. <https://repositorio.uniceub.br/jspui/bitstream/235/7153/1/21115622.pdf

Amaral, L., et al. (2015). Fatores que influenciam na interrupção do aleitamento materno exclusivo em nutrizes. Revista Gaúcha de Enfermagem, 36, 127-134. < https://www.scielo.br/pdf/rgenf/v36nspe/0102-6933-rgenf-36-spe-0127.pdf>.

Aparício, G. (2016). Ajudar a desenvolver hábitos alimentares saudáveis na infância. Centro de Estudos e Tecnologia. <https://revistas.rcaap.pt/millenium/article/view/8263>.

Barros, L. A Desnutrição Infantil e sua Relação com a Rede Social e a Segurança Alimentar e Nutricional da Família. Dissertação (mestrado em nutrição) Universidade Federal de Alagoas, Maceió. <http://200.17.114.109/bitstream/riufal/7306/3/A\%20desnutrição \%20infantil\%20e\%20sua\%20relação\% 20com\%20a\%20rede\%20social\%20e\%20a\%20segurança\%20alimentar\%20e\%20nutricional\%20da\%20família.pdf >.

Bastos, J., et al. (2019). Alimentação saudável no ensino infantil: perspectiva da enfermagem. Rev Inic Cient e Ext., 2(3), 125-130. <https://revistasfacesa.senaaires.com.br/index.php/iniciacao-cientifica/article/view/246/186>.

Bertoletti, J., \& Garcia-Santos, S. C. (2012). Avaliação do Estresse na Obesidade Infantil. PSICO, 43(1), 32-38, < https://revistaseletronicas.pucrs.br/index.p hp/revistapsico/article/view/11091/7616>.

Cabral, P., et al. Motivos do sucesso da amamentação exclusiva na perspectiva dos pais. Revista eletrônica de enfermagem, 15(2), 454-462, 2013. < https://www.revistas.ufg.br/fen/article/view/16996/14824>.

Carvalho, E. et al. (2013). Obesidade: Aspectos Epidemiológicos E Prevenção. Rev Med, 23(1), 74-82. <http://rmmg.org/exportar-pdf/13/v23n1a12.pdf>.

Chagas, D., et al. (2013). Prevalência e fatores associados à desnutrição e ao excesso de peso em menores de cinco anos nos seis maiores municípios do Maranhão. Revista Brasileira Epidemiol, 16(1), 146-156, <https://www.scielosp.org/article/ssm/conten t/raw/?resource_ssm_path=/media/assets/rbe $\mathrm{pid} / \mathrm{v} 16 \mathrm{n} 1 / 1415-790 \mathrm{X}-\mathrm{rbepid}-16-01-0146 . \mathrm{pdf}>$.

Coelho, H., \& Pires, A. (2014). Relações Familiares e Comportamento Alimentar. Psic.: Teor. e Pesq., 30(1), 45-52. < https://www.scielo.br /pdf/ptp/v30n1/06.pdf >.

Cunha, L. (2014). A Importância de uma Alimentação Adequada na Educação Infantil. Monografia (pós-graduação em Ensino de Ciências) - Universidade Tecnológica Federal do Paraná (UTFPR), Universidade de Ibaiti. <http://repositorio.utfpr.edu.br/jspui/bitstream/1/21702/2/MD_ENSCIE_IV_2014_57.pdf>.

Dantas, R., |\& Silva, G. (2019). O Papel Do Ambiente Obesogênico E Dos Estilos De Vida Parentais No Comportamento Alimentar Infantil. Rev Paul Pediatr, 37(3), 363-371 <https://www.scielo.br/pdf/rpp/v37n3/pt_0103-0582-rpp-2019-37-3-00005.pdf〉.

Ferreira, C. (2018). A Importância De Uma Alimentação Adequada na Infância. Monografia (pós-graduação em Ensino de Ciências) - Universidade

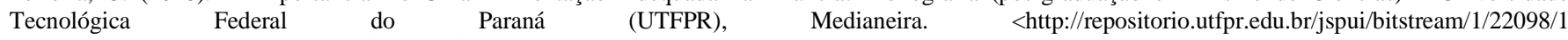
/importanciaalimentacaoadequadainfancia.pdf $>$.

Ferreira, C. (2018). A Importância De Uma Alimentação Adequada na Infância. Monografia (pós-graduação em Ensino de Ciências) - Universidade Tecnológica Federal do Paraná (UTFPR), Medianeira. <http://repositorio.utfpr.edu.br/jspui/bitstream/1/2209 8/1/importancia alimentacaoadeq uadainfancia.pdf>.

Figueiredo, M., et al. (2015). Consulta De Nutrição Na Primeira Infância: Uma Proposta De Protocolo De Atendimento. Nutrire, 40(2), 162-172. < http://sban.cloudpainel.com.br/files/revistas_publicacoes/462.pdf $>$.

Fraga, J., et al. (2012). A Relação Entre A Desnutrição e o Desenvolvimento Infantil. Rev. Assoc. Bras. Nutr., 4(5), 60-62. <https://rasbran.emnuvens.com.br/rasbran/article/view/129/105>.

Frota M. A. et al. Crianças Desnutridas: Percepção Da Família Quanto Ao Cuidado. Cienc Cuid Saude, 10(2), 233-239, 2011. Disponível em: <https://periodicos.uem.br/ojs/index.php/CiencCuidSaude/article/view/10007/pdf>.

Garcia, L., \& Roncalli. (2020). A Determinantes Socioeconômicos E De Saúde Da Desnutrição Infantil: Uma Análise Da Distribuição Espacial. Saúde e pesquisa, 13(3), 595-606. < https://periodicos.unicesumar.edu.br/index.php/saudpesq/article/view/7739/6379>.

Haack, A., \& Martins, M. (2013). Conhecimentos maternos: influência na introdução da alimentação complementar. Com. Ciência Saúde, 23(3), 263-270. <http://bvsms.saude.gov.br/bvs/periodicos/revista_ESCS_v23_n3_a8_conhecimentos_maternos_influencia.pdf >.

Jaime, P. C., et al. (2016). Assistência em saúde e alimentação não saudável em crianças menores de dois anos: dados da Pesquisa Nacional de Saúde, Brasil, Rev. Bras. Saúde Matern. Infant., 16(2), 159-167. <www.scielo.br/pdf/rbsmi/v16n2/pt_1519-3829-rbsmi-16-02-0149.pdf>.

Lima, A. P. E., Javorski, M., \& Vasconcelos, M. G. L. (2011). Práticas alimentares no primeiro ano de vida. Revista Brasileira Enfermagem, 64(5), 912-918, <https://www.scielo.br/pdf/reben/v64n5/a17v64n5.pdf>.

Lima, C., et al. (2019). Auto eficácia na amamentação exclusiva: avaliação dos domínios técnica e pensamentos intrapessoais em puérperas. Enfermagem em foco, 10(3), 9-14. < http://revista.cofen.gov.br/index.php/enfermagem/article/view/1597/539>.

Lima, D., et al. (2012). Alimentação na primeira infância no Brasil. Revista APS, 15(3), 336-344. < https://periodicos.uf jf.br/index.php/aps/article/view/14436/7788>. 
Research, Society and Development, v. 10, n. 15, e238101522901, 2021

(CC BY 4.0) | ISSN 2525-3409 | DOI: http://dx.doi.org/10.33448/rsd-v10i15.22901

Lopes, W., et al. (2018). Alimentação De Crianças Nos Primeiros dois Anos De Vida. Rev Paul Pediatr, 36(2), 164-170. <https://www.scielo.br/pdf/rpp/v36n2/0103-0582-rpp-36-02-164.pdf>

Madruga, W., et al. (2012). Manutenção dos Padrões Alimentares da Infância à Adolescência. Revista Saúde Pública, 46(2), 376-386. <www.scielo.br/pdf/rsp/v46n2/3433.pdf >.

Maranhão, H., et al. (2018). Dificuldades Alimentares em pré-Escolares, Práticas Alimentares pregressas e Estado Nutricional. Rev Paul Pediatr., 36(1), 45-51, <www.scielo.br/pdf/rpp/v36n1/0103-0582-rpp-2018-36-1-00004.pdf>.

Martins, M., \& Santana, L. (2013). Benefícios da amamentação para saúde materna. Interfaces Científicas - Saúde e Ambiente, 1(3), 87-97. <https://periodicos.set.edu.br/saude/article/view/763/443>.

Melo, K. M., et al. (2017). Influência do comportamento dos pais durante a refeição e no excesso de peso na infância Escola Anna Nery. Revista de Enfermagem, 21(4), 1-6. < https://www.redalyc.org/pdf/1277/127752022027.pdf>.

Nascimento, G., et al. (2015). Obesidade Infantil: Causas e Consequências. Eletronic Journal of Pharmacy, 12, 27-28. $\langle$ https://www.revistas.ufg.br/REF/article/view/40824/pdf >.

Oliveira, M., et al. (2017). A importância nutricional da alimentação complementar. Revista Ciências Nutricionais Online, 1(1), 36-45. < https://www.unifafibe.com.br/revistasonline/arquivos/cienciasnutricionaisonline/sumario/46/27032017152234.pdf>.

Oliveira, T., et al. (2017). Associação entre o aleitamento materno, introdução alimentar e desenvolvimento neuropsicomotor nos primeiros seis meses de vida. Distúrb Comun, 29(2), 262-273. <www.researchgate.net/profile/Rodrigo-Carmo/publication/318213608_Associacao_entre_ o_aleitamento_materno_introducao_alimentar_e_desenvolvimento_neuropsicomotor_nos_primeiros_seis_meses_de_vida/links/5c084ad992851c39ebd615a9/ Associacao-entre-o-aleitamento-materno-introducao-alimentar-e-desenvolvimento-neuropsicomotor-nos-primeiros-seis-meses-de-vida.pdf>

Pereira, P., \& Lopes, L. (2012). Obesidade Infantil: Estudo em Crianças Num ATL. 42, 105. -125<https://revistas.rcaap.pt/millenium/article/view/8197/5807>.

Rosa, J., Delgado, S. (2017). Conhecimento de puérperas sobre amamentação e introdução alimentar. Revista Brasileira em promoção da saúde, 30(4), 1-9, $\langle$ http://periodicos.unifor.br/RBPS/article/view/6199>.

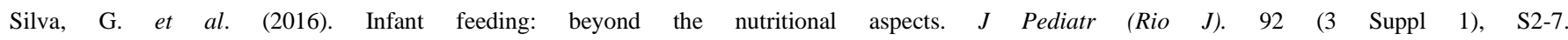
〈https://www.scielo.br/pdf/jped/v92n3s1/pt_0021-7557-jped-92-03-s1-00S2.pdf〉.

Soares, L. et al. (2013-2014). A Transição da Desnutrição Para a Obesidade. Braz. J. Surg. Clin. Res, 5(1), 64-68. <https://www.mastereditora.com.br/periodico/20140429_2131532.pdf\#page=64>.

Tenorio, A., Cobayashi, F. (2011). Obesidade Infantil na Percepção dos Pais. Rev Paul Pediatr, 29(4), 634-639, $<$ https://www.scielo.br/pdf/rpp/v29n4/25.pdf>.

Toloni, M., et al. (2011). Introdução de alimentos industrializados e de alimentos de uso tradicional na dieta de crianças de creches públicas no município de São Paulo. Revista de nutrição, 24(1), 61-70. <https://www.scielo.br/pdf/rn/v24n1/v24n1a06.pdf>.

Vicari, E. (2013). Aleitamento materno, a introdução da alimentação complementar e sua relação com a obesidade infantil. Revista Brasileira de Obesidade, Nutrição e Emagrecimento, 7(40), 72-83. <http://www.rbone.com.br/index.php/rbone/article/view/306>.

Victorino, S., et al. (2014). Viver Com Obesidade Infantil: A Experiência De Crianças Inscritas Em Programa De Acompanhamento Multidisciplinar. Rev Rene., 15(6), 980-989. <http://periodicos.ufc.br/rene/article/view/3295/2534>. 